\title{
Numerical Solutions of Fourth-Order Fractional Integro-Differential Equations
}

\author{
Ahmet Yıldırım, Sefa Anıl Sezer, and Yasemin Kaplan \\ Ege University, Department of Mathematics, 35100 Bornova-İzmir, Turkey \\ Reprint requests to A. Y.; E-mail: ahmet.yildirim@ege.edu.tr or ahmetyildirim80@gmail.com \\ Z. Naturforsch. 65a, 1027 - 1032 (2010); received November 16, 2009
}

\begin{abstract}
In this paper, the homotopy perturbation method (HPM) is developed to obtain numerical solutions of linear and nonlinear boundary value problems for fourth-order fractional integro-differential equations. The fractional derivatives are described in the Caputo sense. Some examples are given and comparisons are made; the comparisons show that the homotopy perturbation method is very effective and convenient and overcome the difficulty of traditional methods. Numerical examples are presented to illustrate the efficiency, simplicity, and reliability of the method.
\end{abstract}

Key words: Homotopy Perturbation Method; Boundary Value Problems; Integro-Differential Equations; Fractional Derivative; Caputo Sense.

\section{Introduction}

In recent years, considerable interest in fractional differential equations has been stimulated due to their numerous applications in the areas of physics and engineering. Differential equations involving derivatives of non-integer order have shown to be adequate models for various physical phenomena in areas like rheology, damping laws, diffusion processes, etc. [1-4]. This is more realistic and it is one reason why fractional calculus has become more and more popular.

Most fractional differential equations do not have exact analytic solutions, so approximation and numerical techniques must be used. Only approximate solutions can be derived using a linearization or perturbation method. For differential equations of integer order, the explicit and numerical solution has also for a long time been a standard topic in physics and mathematics. No analytical method was available before 1998 for such equations, even for linear fractional differential equations. In 1998, the variational iteration method (VIM) was first proposed to solve fractional differential equations with greatest success [4]. Many authors found VIM as an effective way to solve fractional equations, both linear and nonlinear [5,6]. Momani and Odibat [7], Ganji et al. [8], and Yıldırım [9,10] applied the homotopy perturbation method (HPM) to fractional differential equations and revealed that HPM is an alternative analytical method for solving fractional differential equations. Momani and Odibat [11] and Odibat and Momani [12] compared the solution procedure between VIM and HPM. According to the fractal spacetime theory (El-Naschie's e-infinity theory), time and space are discontinuous, and the fractional model is the best candidate to describe such problems. Time-fractional equations always behave fascinatingly as illustrated in $[13,14]$.

In this paper, the HPM is implemented to derive analytic approximate solutions to linear and nonlinear boundary value problems for fourth-order fractional integro-differential equations. Recently, Momani and Noor [15] used the Adomian decomposition method for solving the governing problem.

The homotopy perturbation method was first proposed by the Chinese mathematician Ji-Huan He [1619]. The essential idea of this method is to introduce a homotopy parameter, say $p$, which takes values from 0 to 1 . When $p=0$, the system of equations usually reduces to a sufficiently simplied form, which normally admits a rather simple solution. As $p$ gradually increases to 1 , the system goes through a sequence of deformations, the solution for each of which is close to that at the previous stage of deformation. Finally at $p=1$, the system takes the original form of the equation and the final stage of deformation gives the desired solution. One of the most remarkable features of the HPM is that usually just few perturbation terms are sufficient for obtaining a reasonably accurate solution. Considerable research works have been conducted recently in applying this method to a class of 
linear and nonlinear equations [20-29]. The interested reader can see [30 - 33] for the latest state of the development of HPM.

There are several definitions of a fractional derivative of order $\alpha>0$ [3,34-37]. The two most commonly used definitions are the Riemann-Liouville and the Caputo. Each definition uses Riemann-Liouville fractional integration and derivatives of whole order. The Riemann-Liouville fractional integration of order $\alpha$ is defined as

$$
\mathbf{J}_{a}^{\alpha} f(x)=\frac{1}{\Gamma(\alpha)} \int_{a}^{x}(x-t)^{a-1} f(t) \mathrm{d} t, \quad x>0,
$$

and the Caputo fractional derivative of order $\alpha$ is defined as

$$
\mathrm{D}_{a}^{\alpha} f(x)=\mathrm{J}_{a}^{m-\alpha} \mathrm{D}^{m} f(x) .
$$

The Caputo fractional derivatives are considered here because it allows traditional initial and boundary conditions to be included in the formulation of the problem. In this paper, we consider the fourth-order fractional integro-differential equations, and the fractional derivatives are taken in the Caputo sense as follows.

Definition 1.1. For $m$ to be the smallest integer that exceeds $\alpha$, the Caputo time-fractional derivative operator of order $\alpha>0$ is defined as

$$
\begin{aligned}
& \mathrm{D}_{t}^{\alpha} u(x, t)=\frac{\partial^{\alpha} u(x, t)}{\partial t^{\alpha}} \\
& =\left\{\begin{array}{l}
\frac{1}{\Gamma(m-\alpha)} \int_{0}^{t}(t-\tau)^{m-\alpha-1} \frac{\partial^{\prime \prime \prime} u(x, t)}{\partial t^{m}} \\
\text { for } m-1<\alpha<m, \\
\frac{\partial^{m} u(x, t)}{\partial t^{m}} \quad \text { for } \alpha=m \in \mathbb{N} .
\end{array}\right.
\end{aligned}
$$

For more information on the mathematical properties of fractional derivatives and integrals one can consult the mentioned references.

The paper is organized as follows. In Section 2, we extend the application of the homotopy perturbation method to construct our numerical solutions for the fourth-order fractional integro-differential equations. In Section 3, we present two examples to show the efficiency and simplicity of the method.

\section{HPM Solutions of Fourth-Order Fractional Integro-Differential Equations}

Consider the boundary value problem for the fourthorder fractional integro-differential equations

$$
\begin{aligned}
& \frac{\partial^{\alpha} y(x)}{\partial x^{\alpha}}= f(x)+\gamma y(x) \\
&+\int_{0}^{x}[g(t) y(t)+h(t) F(y(t))] \mathrm{d} t, \\
& 0<x<b, \quad 3<\alpha \leq 4,
\end{aligned}
$$

subject to the boundary conditions

$$
\begin{aligned}
& y(0)=\gamma_{0}, \quad y^{\prime \prime}(0)=\gamma_{2}, \\
& y(b)=\beta_{0}, \quad y^{\prime \prime}(b)=\beta_{2} .
\end{aligned}
$$

The standard form of the fourth-order fractional integro-differential equations (1) can be written in terms of operator forms as

$$
\begin{aligned}
& \mathrm{D}_{x}^{\alpha} y(x)= f(x)+\gamma y(x) \\
&+\int_{0}^{x}[g(t) y(t)+h(t) F(y(t))] \mathrm{d} t, \\
& 0<x<b, \quad 3<\alpha \leq 4,
\end{aligned}
$$

where $\mathrm{D}_{x}^{\alpha}$ indicates the $\alpha$ th Caputo fractional derivative, and $F(y(x))$ is a nonlinear continuous function, $\gamma, \gamma_{0}, \gamma_{2}, \beta_{0}$, and $\beta_{2}$ are real constants, $f, g$, and $h$ are given functions that can be approximated by Taylor polynomials. Also, in this paper we use the inverse operator $\mathrm{J}^{\alpha}$ of $\mathrm{D}_{x}^{\alpha}$, then the boundary conditions are used. The general response expression contains a parameter describing the order of the fractional derivatives that can be varied to obtain various responses. In the case of $\alpha=4$, the fractional equation reduces to the classical fourth-order integro-differential equation.

To solve (4) by the homotopy perturbation method, we construct the following homotopy:

$$
\begin{aligned}
& \mathrm{D}_{x}^{\alpha} y(x)= \\
& p\left[\gamma y(x)+\int_{0}^{x}[g(t) y(t)+h(t) F(y(t))] \mathrm{d} t\right]+f(x) .
\end{aligned}
$$

In view of HPM, we use the homotopy parameter $p$ to expand the solution

$$
y=y_{0}+p y_{1}+p^{2} y_{2}+p^{3} y_{3}+\ldots .
$$

Substituting (6) into (5) and equating the coefficients of like powers of $p$, we get the following set of fractional differential equations:

$$
\begin{aligned}
& p^{0}: \mathrm{D}_{x}^{\alpha} y_{0}=f(x), \\
& p^{1}: \mathrm{D}_{x}^{\alpha} y_{1}=\gamma y_{0}(x)+\int_{0}^{x}\left[g(t) y_{0}(t)+h(t) A_{0}\right] \mathrm{d} t, \\
& p^{2}: \mathrm{D}_{x}^{\alpha} y_{2}=\gamma y_{1}(x)+\int_{0}^{x}\left[g(t) y_{1}(t)+h(t) A_{1}\right] \mathrm{d} t,
\end{aligned}
$$


$p^{3}: \mathrm{D}_{x}^{\alpha} y_{3}=\gamma y_{2}(x)+\int_{0}^{x}\left[g(t) y_{2}(t)+h(t) A_{2}\right] \mathrm{d} t$,

$p^{n+1}: \mathrm{D}_{x}^{\alpha} y_{n}=\gamma y_{n}(x)+\int_{0}^{x}\left[g(t) y_{n}(t)+h(t) A_{n}\right] \mathrm{d} t,(11$

Applying the inverse operator $\mathrm{J}_{\alpha}$ of $\mathrm{D}_{x}^{\alpha}$ to both sides of (7) - (11), the iterates are determined by the following recursive way:

$$
y_{0}(x)=\gamma_{0}+\gamma_{1} x+\gamma_{2} \frac{x^{2}}{2 !}+\gamma_{3} \frac{x^{3}}{3 !}+\mathbf{J}^{\alpha} f(x),
$$

$y_{n+1}(x)=\gamma \mathbf{J}^{\alpha} y_{n}(x)+\mathbf{J}^{\alpha}\left(\int_{0}^{x}\left[g(t) y_{n}(t)+h(t) A_{n}\right] \mathrm{d} t\right)$,

where

$$
F(y(t))=\sum_{n=0}^{\infty} p^{n} A_{n}
$$

and $\gamma_{1}=y^{\prime}(0)$ and $\gamma_{3}=y^{\prime \prime \prime}(0)$ are to be determined.

The HPM series solutions generally converge very rapidly. For later numerical computation, let the expression

$$
\phi_{N}(x)=\sum_{n=0}^{N-1} y_{n}(x)
$$

denote the $N$-term approximation to $y(x)$. We note that at this stage $\gamma_{1}$ and $\gamma_{3}$ are still undetermined. Now imposing the boundary conditions (3) on (15) gives the system

$$
\begin{array}{r}
\phi_{N}(b)=y_{0}(b)+y_{1}(b)+\ldots+y_{N-1}(b)=\beta_{0}, \\
\phi_{N}^{\prime \prime}(b)=y_{0}^{\prime \prime}(b)+y_{1}^{\prime \prime}(b)+\ldots+y_{N-1}^{\prime \prime}(b)=\beta_{2},
\end{array}
$$

from which the unknowns $\gamma_{1}$ and $\gamma_{3}$ can be found. Once we have the values for $\gamma_{1}$ and $\gamma_{3}$, expression (15) serves as an approximate solution of (1) - (3).

\section{Examples}

To illustrate the solution procedure and show the feasibility and efficiency of the HPM, we have applied the method to two different examples with known exact solution for the special case $\alpha=4$. All the results are calculated by using the symbolic computation software Maple.

\subsection{Example 1}

At first,we consider the following linear fourthorder fractional integro-differential equation [15]:

$$
\begin{aligned}
& \mathrm{D}_{x}^{\alpha} y(x)=x\left(1+\mathrm{e}^{x}\right)+3 \mathrm{e}^{x}+y(x)-\int_{0}^{x} y(t) \mathrm{d} t, \\
& 0<x<1, \quad 3<\alpha \leq 4
\end{aligned}
$$

subject to the boundary conditions

$$
\begin{aligned}
& y(0)=1, \quad y^{\prime \prime}(0)=2, \\
& y(1)=1+\mathrm{e}, \quad y^{\prime \prime}(1)=3 \mathrm{e} .
\end{aligned}
$$

The exact solution of (18), for the special case $\alpha=4$, is given by

$$
y(x)=1+x \mathrm{e}^{x} .
$$

According to the homotopy perturbation procedures (1) - (17) we now successively obtain the recursive homotopy perturbation algorithm

$$
\begin{aligned}
& y_{0}(x)=1+A x+x^{2}+\frac{1}{3 !} B x^{3}+\mathrm{J}^{\alpha}\left(x+x \mathrm{e}^{x}+3 \mathrm{e}^{x}\right), \\
& y_{n+1}(x)=\mathrm{J}^{\alpha} y_{n}(x)-\mathrm{J}^{\alpha}\left(\int_{0}^{x} y_{n}(t) \mathrm{d} t\right), n \geq 0,
\end{aligned}
$$

where the constants $A=y^{\prime}(0)$ and $B=y^{\prime \prime \prime}(0)$ are to be determined.

In order to avoid difficult fractional integration and to show the efficiency of the present method for solving fractional integro-differential equations, we can simplify the integrations by taking the truncated Taylor expansions for the exponential term in (22):

$$
\mathrm{e}^{x} \approx 1+x+\frac{x^{2}}{2}+\frac{x^{3}}{6}
$$

Using the iterative algorithm (22) yields the 2-term approximation

$$
\begin{aligned}
& \phi_{2}(x)=y_{0}(x)+y_{1}(x)=1+A x+x^{2}+\frac{1}{3 !} B x^{3} \\
& \left.+\mathrm{J}^{\alpha}\left[x+(x+3)\left(1+x+\frac{x^{2}}{2}+\frac{x^{3}}{6}\right)\right]\right)+\mathrm{J}^{\alpha} y_{0}(x) \\
& -\mathrm{J}^{\alpha}\left(\int_{0}^{x} y_{0}(t) \mathrm{d} t\right)=1+A x+x^{2}+\frac{B x^{3}}{6}+\frac{4 x^{\alpha}}{\alpha \Gamma(\alpha)} \\
& -\frac{x^{1+\alpha}}{\left(\alpha+\alpha^{2}\right) \Gamma(\alpha)}+\frac{5 x^{1+\alpha}}{\left(2+3 \alpha+\alpha^{2}\right) \Gamma(\alpha)} \\
& +\frac{A x^{1+\alpha}}{\left(2+3 \alpha+\alpha^{2}\right) \Gamma(\alpha)}+\frac{10 x^{1+\alpha}}{\alpha\left(2+3 \alpha+\alpha^{2}\right) \Gamma(\alpha)}
\end{aligned}
$$




\begin{tabular}{lllll}
\hline & $\alpha=3.25$ & $\alpha=3.5$ & $\alpha=3.75$ & $\alpha=4$ \\
\hline$A$ & 1.1001563039108 & 1.0909836873321 & 1.0518681277001 & 0.998909525423 \\
$B$ & -0.2814228833424 & 0.9726301531206 & 2.0741898151992 & 3.0073098796724 \\
\hline & & & & \\
\hline & $\alpha=3.25$ & $\alpha=3.5$ & $\alpha=3.75$ & $\alpha=4$ \\
\hline 0.0 & 1.0 & 1.0 & 1.0 & 1.0 \\
0.1 & 1.1202485492579 & 1.1193723618931 & 1.1155765608041 & 1.1104092625522 \\
0.2 & 1.2624009459051 & 1.2607965228726 & 1.2537481910102 & 1.2440722001297 \\
0.3 & 1.4293559759369 & 1.4272175665922 & 1.4177578632416 & 1.4046633667259 \\
0.4 & 1.628578688995 & 1.6224035710616 & 1.6115327558068 & 1.5963715062378 \\
0.5 & 1.8534779619798 & 1.8509379570966 & 1.8397180005463 & 1.823670094191 \\
0.6 & 2.1206550639506 & 2.1182600923197 & 2.1077351456080 & 2.0928776743143 \\
0.7 & 2.4327620675062 & 2.4307302105896 & 2.4218566791608 & 2.4092736235270 \\
0.8 & 2.7971904644190 & 2.7957119076815 & 2.7892942668210 & 2.7801622797210 \\
0.9 & 3.2224499000323 & 3.2216698320246 & 3.2183001428505 & 3.2134943508078 \\
1.0 & 3.7182818284590 & 3.7182818284590 & 3.7182818284590 & 3.7182818284590 \\
\hline
\end{tabular}

Table 1. Values of the constants $A$ and $B$ for different values of $\alpha$ using (23).

$+\frac{2 A x^{1+\alpha}}{\alpha\left(2+3 \alpha+\alpha^{2}\right) \Gamma(\alpha)}+\frac{5 x^{2+\alpha}}{\alpha\left(2+3 \alpha+\alpha^{2}\right) \Gamma(\alpha)}$

$-\frac{A x^{2+\alpha}}{\alpha\left(2+3 \alpha+\alpha^{2}\right) \Gamma(\alpha)}+\frac{2 x^{2+\alpha}}{\left(2 \alpha+3 \alpha^{2}+\alpha^{3}\right) \Gamma(\alpha)}$

$+\frac{3 \sqrt{\pi} x^{2 \alpha}}{4^{\alpha} \Gamma(\alpha) \Gamma\left(\frac{1}{2}+\alpha\right)}+\frac{4 x^{3+\alpha}}{\Gamma(4+\alpha)}+\frac{B x^{3+\alpha}}{\Gamma(4+\alpha)}$

$+\frac{4 x^{4+\alpha}}{\Gamma(5+\alpha)}-\frac{B x^{4+\alpha}}{\Gamma(5+\alpha)}-\frac{3 x^{1+2 \alpha}}{\Gamma(2+2 \alpha)}$

$+\frac{10 x^{1+2 \alpha}}{(2+\alpha) \Gamma(2+2 \alpha)}+\frac{5 x^{1+2 \alpha} \Gamma(1+\alpha)}{(2+\alpha) \Gamma(\alpha) \Gamma(2+2 \alpha)}$

$+\frac{5 x^{2+2 \alpha}}{\Gamma(3+2 \alpha)}-\frac{10 x^{2+2 \alpha}}{(2+\alpha) \Gamma(3+2 \alpha)}$

$-\frac{5 x^{2+2 \alpha} \Gamma(1+\alpha)}{(2+\alpha) \Gamma(\alpha) \Gamma(3+2 \alpha)}+\frac{x^{3+2 \alpha}}{\Gamma(4+2 \alpha)}$

$-\frac{2 x^{4+2 \alpha}}{\Gamma(5+2 \alpha)}-\frac{4 x^{5+2 \alpha}}{\Gamma(6+2 \alpha)}$,

where $A$ and $B$ can be determined using the boundary conditions (20).

Table 1 shows the values of $A$ and $B$ for different values of $\alpha$. Setting $\alpha=4$ in (23), we reproduce the solution of [38] for the classical integro-differential equation.

Table 2 shows the solution for different values of $\alpha$. The value of $\alpha=4$ is the only case for which we know the exact solution and our approximate solution is in good agreement with the exact values. It is evident that the efficiency of this approach can be dramatically enhanced by computing further terms of approximate solutions and/or by taking more terms in the Taylor expansion for the exponential term.

The comparison of the exact solution (21) and the approximate solution (23) obtained using the homo-

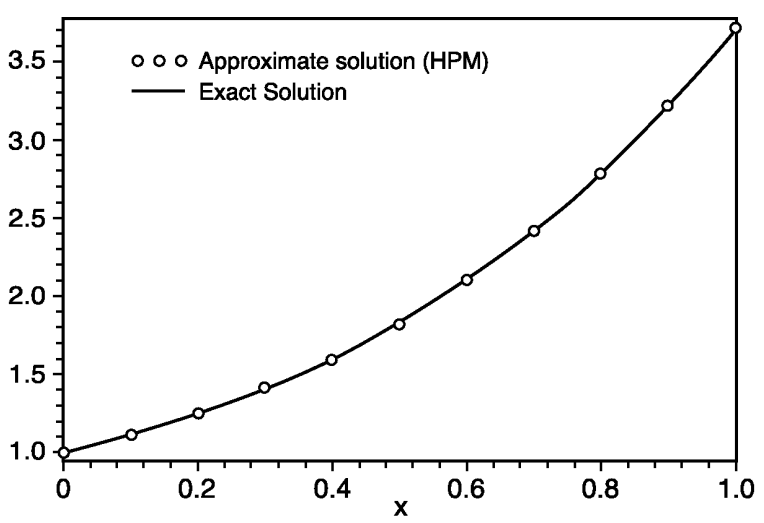

Fig. 1. Comparison of the exact solution (21) and the approximate solution (23) for $\alpha=4$.

topy perturbation method, for the special case $\alpha=4$ is shown in Figure 1. It can be seen from this figure that the solution obtained by the present method is identical with the exact solution.

\subsection{Example 2}

Next, we consider the following nonlinear fourthorder fractional integro-differential equation [15]:

$$
\begin{aligned}
& \mathrm{D}_{x}^{\alpha} y(x)=1+\int_{0}^{x} \mathrm{e}^{-t} y^{2}(t) \mathrm{d} t, \\
& 0<x<1, \quad 3<\alpha \leq 4,
\end{aligned}
$$

subject to the boundary conditions

$$
\begin{aligned}
& y(0)=1, \quad y^{\prime \prime}(0)=1, \\
& y(1)=\mathrm{e}, \quad y^{\prime \prime}(1)=\mathrm{e} .
\end{aligned}
$$




\begin{tabular}{lllll}
\hline & $\alpha=3.25$ & $\alpha=3.5$ & $\alpha=3.75$ & $\alpha=4$ \\
\hline$A$ & 1.0143373569096 & 1.0163004456602 & 1.0100788445491 & 0.9998909026929 \\
$B$ & 0.2902766560668 & 0.5565465660885 & 0.7957407595086 & 1.0007021517774 \\
\hline & & & & \\
\hline & $\alpha=3.25$ & $\alpha=3.5$ & $\alpha=3.75$ & $\alpha=4$ \\
\hline 0.0 & 1.0 & 1.0 & 1.0 & 1.0 \\
0.1 & 1.1065516178821 & 1.10675060464047 & 1.1061514591278 & 1.1051601253699 \\
0.2 & 1.2239318517036 & 1.22432393215707 & 1.2232273020046 & 1.2213818748800 \\
0.3 & 1.3532000130443 & 1.35375573773717 & 1.3523083486232 & 1.3498292377736 \\
0.4 & 1.4956008613929 & 1.49627053696334 & 1.4946359532222 & 1.4917885460168 \\
0.5 & 1.6525535166189 & 1.65327350718537 & 1.6516152073248 & 1.6486813365191 \\
0.6 & 1.8256546719897 & 1.82635407419518 & 1.8248237325163 & 1.8220785540392 \\
0.7 & 2.0166875976845 & 2.01729489459862 & 2.0160237167113 & 2.0137162158054 \\
0.8 & 2.2276344843814 & 2.22808428028207 & 2.2271764206100 & 2.2255126505348 \\
0.9 & 2.4606909505605 & 2.46093114391032 & 2.4604587805248 & 2.4595874006832 \\
1.0 & 2.7182818284590 & 2.71828182845904 & 2.7182818284590 & 2.7182818284590 \\
\hline
\end{tabular}

Table 3. Values of the constants $A$ and $B$ for different values of $\alpha$ using (30).

The exact solution of (24), for the special case $\alpha=4$, is given by

$$
y(x)=\mathrm{e}^{x} .
$$

According to the homotopy perturbation procedures (1) - (17) we now successively obtain the recursive homotopy perturbation algorithm

$$
\begin{aligned}
& y_{0}(x)=1+A x+x^{2}+\frac{1}{3 !} B x^{3}+\mathrm{J}^{\alpha}(1), \\
& y_{n+1}(x)=\mathrm{J}^{\alpha}\left(\int_{0}^{x} \mathrm{e}^{-t} A_{n}(t) \mathrm{d} t\right), \quad n \geq 0,
\end{aligned}
$$

where

$$
A_{n}(t)=\sum_{i=0}^{n} y_{i}(t) y_{n-i}(t)
$$

and the constants $A=y^{\prime}(0)$ and $B=y^{\prime \prime \prime}(0)$ are to be determined.

In order to avoid the difficult fractional integration and to show the efficiency of the present method for solving fractional integro-differential equations, we can simplify the integrations by taking the truncated Taylor expansions for the exponential term in (28):

$$
\mathrm{e}^{-x} \approx 1-x+\frac{x^{2}}{2}-\frac{x^{3}}{6}
$$

Using the recursive algorithm (28) and (29) to calculate the polynomials for the nonlinear term $F(y)=y^{2}$, we obtain the 2-term approximation

$$
\begin{aligned}
& \phi_{2}(x)=y_{0}(x)+y_{1}(x)=1+A x+x^{2}+\frac{B x^{3}}{6}+\frac{x^{\alpha}}{\alpha \Gamma(\alpha)} \\
& +\mathbf{J}^{\alpha}\left[\int_{0}^{x}\left(1-t+\frac{t^{2}}{2}-\frac{t^{3}}{6}\right) A_{0}(t) \mathrm{d} t\right],
\end{aligned}
$$

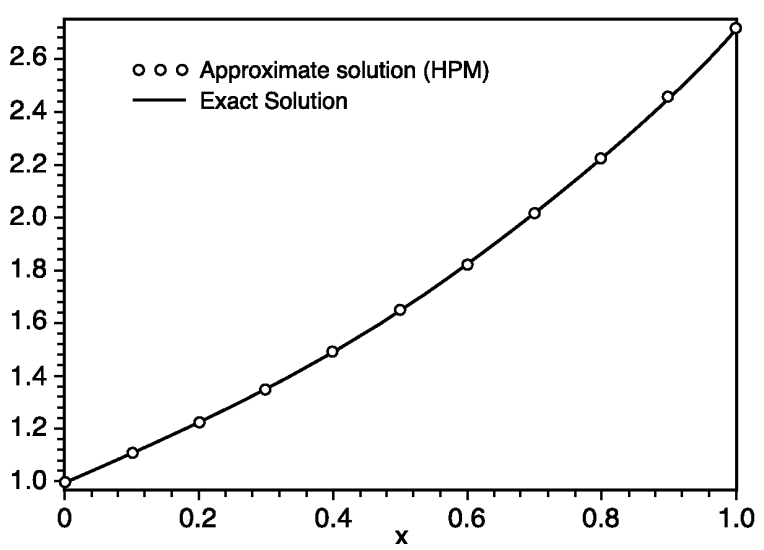

Fig. 2. Comparison of the exact solution (27) and the approximate solution (30) for $\alpha=4$.

where $A$ and $B$ can be determined using the boundary conditions (26).

Table 3 shows the values of $A$ and $B$ for different values of $\alpha$. Setting $\alpha=4$ in (30), we reproduce the solution of [38] for the classical integro-differential equation.

Table 4 shows the solution for different values of $\alpha$. The value of $\alpha=4$ is the only case for which we know the exact solution and our approximate solution is in good agreement with the exact values. It is evident that the efficiency of this approach can be dramatically enhanced by computing further terms of the approximate solution and/or by taking more terms in the Taylor expansion for the exponential term.

The comparison of the exact solution (27) and the approximate solution (30) obtained using the homotopy perturbation method for the special case $\alpha=4$ is shown in Figure 2. It can be seen from this figure that 
the solution obtained by the present method is identical with the exact solution.

\section{Conclusion}

In this paper, we used the homotopy perturbation method for finding the solution of linear and nonlinear boundary value problems for fourth-order fractional integro-differential equations. The method was

[1] B. J. West, M. Bolognab, and P. Grigolini, Physics of Fractal Operators, Springer, New York 2003.

[2] A. V. Chechkin, R. Gorenflo, I. M. Sokolov, and V. Yu. Gonchar, Frac. Calc. Appl. Anal. 6, 259 (2003).

[3] I. Podlubny, Fractional Differential Equations, Academic, San Diego 1999.

[4] J. H. He, Comput. Methods Appl. Mech. Eng. 167, 57 (1998).

[5] Z. Odibat and S. Momani, Int. J. Nonlinear Sci. Numer. Simul. 7, 27 (2006).

[6] S. Das, Int. J. Nonlinear Sci. Numer. Simul. 9, 361 (2008).

[7] S. Momani and Z. Odibat, Phys. Lett. A 365, 345 (2007).

[8] Z.Z. Ganji, D. D. Ganji, H. Jafari, and M. Rostamian, Topolog. Methods Nonlinear Anal. 31, 341 (2008).

[9] A. Yıldırım, Int. J. Nonlinear Sci. Numer. Simul. 10, 445 (2009).

[10] A. Yildırım, Int. J. Comput. Math. DOI: 10.1080/ 00207160902874653.

[11] S. Momani, Z. Odibat, and I. Hashim, Topolog. Methods Nonlinear Anal. 31, 211 (2008).

[12] Z. Odibat and S. Momani, Topolog. Methods Nonlinear Anal. 31, 227 (2008).

[13] L.-J. Sheu, L.-M. Tam, and S.-K. Lao, Int. J. Nonlinear Sci. Numer. Simul. 10, 33 (2009).

[14] C. Xu, G. Wu, J. W. Feng, and W. Q. Zhang, Int. J. Nonlinear Sci. Numer. Simul. 9, 89 (2008).

[15] S. Momani and M. A. Noor, Appl. Math. Comput. 182, 754 (2006).

[16] J. H. He, Chaos, Solitons, and Fractals 26, 695 (2005).

[17] J.H. He, Int. J. Nonlinear Sci. Numer. Simul. 6, 207 (2005).

[18] J. H. He, Phys. Lett. A 350, 87 (2006). used in a direct way without using linearization, perturbation or restrictive assumptions. We showed that the HPM is a very efficient and powerful tool in finding the solutions of the fractional differential equations. It provides more realistic series solutions that converge very rapidly in real physical problems. In our paper, we use the Maple Package to calculate the functions obtained from the homotopy perturbation method.

[19] J. H. He, Comput. Methods Appl. Mech. Eng. 178, 257 (1999).

[20] A. Yıldırım and T. Öziş, Phys. Lett. A 369, 70 (2007).

[21] A. Yildırım, Comput. Math. Appl. 56, 3175 (2008).

[22] A. Yıldırım, Int. J. Comput. Math. 87, 992 (2010).

[23] A. Yıldırım, Z. Naturforsch. 63a, 621 (2008).

[24] A. Yıldırım, Commun. Numer. Methods Eng. 26, 1144 (2010).

[25] S. T. Mohyud-Din, M. A. Noor, and K. I. Noor, Int. J. Nonlinear Sci. Numer. Simul. 10, 223 (2009).

[26] F. Shakeri and M. Dehghan, Phys. Scri. 75, 551 (2007).

[27] M. Dehghan and F. Shakeri, Phys. Scri. 75, 778 (2007).

[28] F. Shakeri and M. Dehghan, Math. Comput. Modelling 48, 486 (2008).

[29] M. T. Darvishi, F. Khani, S. Hamedi-Nezhad, and S.W. Ryu, Int. J. Comput. Math. 87, 908 (2010).

[30] J. H. He, Topolog. Methods Nonlinear Anal. 31, 205 (2008).

[31] J. H. He, Int. J. Mod. Phys. B 20, 1141 (2006).

[32] J. H. He, Int. J. Mod. Phys. B 20, 2561 (2006).

[33] J. H. He, Int. J. Mod. Phys. B 22, 3487 (2008).

[34] K. S. Miller and B. Ross, An Introduction to the Fractional Calculus and Fractional Differential Equations, Wiley, New York 1993.

[35] S. G. Samko, A. A. Kilbas, and O. I. Marichev, Fractional Integrals and Derivatives: Theory and Applications, Gordon and Breach, Yverdon 1993.

[36] K. B. Oldham and J. Spanier, The fractional calculus, Academic Press, New York 1974.

[37] Y. Luchko and R. Gorneflo, The initial value problem for some fractional differential equations with the $\mathrm{Ca}$ puto derivative, Preprint series A08-98, Fachbreich Mathematik und Informatik, Freie Universitat Berlin 1998.

[38] I. Hashim, J. Comput. Appl. Math. 193, 658 (2006). 